\title{
La Suisse paie un prix élevé pour les maladies non transmissibles
}

\author{
Carola A. Huber ${ }^{\text {a }}$, Simon Wieser ${ }^{\mathrm{b}}$ \\ a Dr phil., MPH, recherche sur les services de santé, recherche de la santé publique, Helsana \\ b Prof. Dr oec. publ., responsable de l'Institut de l'économie de la santé, Winterthour, Haute école zurichoise des sciences appliquées
}

Qu'il s'agisse de maladies cardio-vasculaires, de cancers ou de maladies respiratoires, les maladies non transmissibles (MNT) représentent aujourd'hui la cause de mortalité la plus importante des femmes et des hommes en Suisse. Une équipe scientifique interdisciplinaire a établi pour la première fois les coûts des maladies non transmissibles de la population en Suisse.

Jusqu'à présent, on savait très peu comment les dépenses de santé en Suisse se répartissaient sur les différentes maladies. Une étude publiée récemment dans le European Journal of Health Economics examine pour la première fois de manière systématique et détaillée les dépenses de santé sous cette perspective et crée de la transparence [1]. Les maladies non transmissibles représentent $80 \%$ des dépenses totales (51 milliards de francs sur 65 en 2011). Ces chiffres ne sont pourtant pas totalement inconnus puisque la publication repose sur un rapport demandé par l'OFSP sur les coûts des mala-

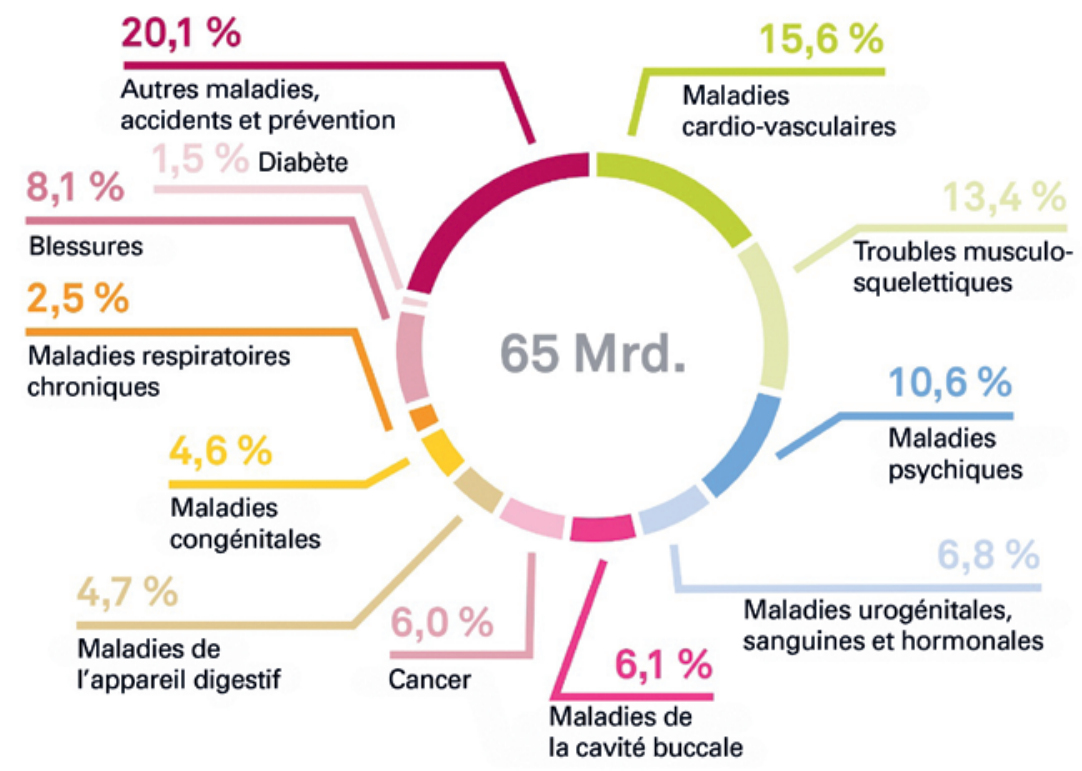

Figure 1: Les dépenses de santé suisses par groupe de maladies. dies non transmissibles, établi en 2014 par la Haute école zurichoise des sciences appliquées, l'Université de Zurich, l'Institut de recherche Polynomics et l'assurance-maladie Helsana.

Les maladies cardio-vasculaires pointent à la première place des affections les plus coûteuses, avec plus de 10 milliards de francs, soit 15,6\% de l'ensemble des coûts de la santé (cf. fig. 1). Elles représentent par ailleurs la cause de mortalité la plus importante des hommes et des femmes (plus de 20000 décès par an) et se rangent à la troisième place des raisons d'hospitalisation les plus fréquentes en Suisse mais aussi dans le monde [2]. En termes de dépenses, les troubles musculo-squelettiques comme l'arthrose, les rhumatismes et les douleurs de dos se placent au deuxième rang, avec 8,7 milliards de francs $(13,4 \%)$, suivis à la troisième place des maladies psychiques, telles que la dépression, qui réunissent près de $10,6 \%$ de l'ensemble des coûts $(6,9$ milliards de francs). Les maladies cancéreuses, souvent évoquées pour leur facteur de coûts, se positionnent à la $7^{\mathrm{e}}$ place $(3,9$ milliards de francs, $6 \%$ ) devant les blessures (5,2 milliards de francs, 8,1\%), les maladies urogénitales, sanguines et hormonales (4,4 milliards de francs, $6,8 \%$ ) et les maladies de la cavité buccale (4 milliards de francs, 6,1\% de l'ensemble des coûts).

\section{L'étude mesure les coûts de traitement directs}

L'étude montre les coûts directs du traitement d'une maladie mais elle ne distingue pas les coûts liés à la prise en charge des affections consécutives à cette maladie. L'exemple du diabète permet de visualiser cette 
différence: le diabète est un facteur de risque important des maladies cardio-vasculaires. Mais les coûts des maladies cardio-vasculaires provoquées par le diabète sont comptabilisés avec les maladies cardiovasculaires et non avec le diabète. Par conséquent, les coûts de traitement induits par le diabète sont de facto nettement plus élevés que les 1,5\% de l'ensemble des coûts répertoriés par l'étude. L'ostéoporose (un trouble musculo-squelettique) amène à la même réflexion: bien que les chutes de patients atteints d'ostéoporose exigent souvent des soins hospitaliers importants, ces dépenses sont attribuées aux blessures et non aux troubles musculo-squelettiques.

\section{Les douleurs dorsales - une maladie très répandue et coûteuse}

Avec une part de 13\% de l'ensemble des dépenses, les troubles musculo-squelettiques se placent en deuxième position des maladies les plus coûteuses. En plus de l'arthrose et de l'arthrite rhumatoïde, ce sont surtout les douleurs de dos qui prévalent. Cela peut paraître étonnant car les traitements contre les douleurs de dos sont communément considérés comme peu chers mais, en revanche, ces douleurs sont très fréquentes. En 2012 par exemple, plus de 40\% des adultes indiquaient avoir souffert du dos au cours des quatre semaines précédant l'enquête (enquête suisse sur la santé [3]).

\section{Troubles mentaux largement répandus}

Les troubles mentaux se classent au troisième rang des maladies les plus coûteuses. S'il est souvent question d'une forte prévalence, le nombre absolu d'antidépresseurs remis reste jusqu'à aujourd'hui inconnu. Le groupe de recherche de la santé publique d'Helsana travaille actuellement avec l'Institut de psychologie de l'Université de Zurich à une nouvelle étude dans le but de dresser pour la première fois un état des lieux détaillé de la prescription d'antidépresseurs en Suisse. Une première analyse/enquête a permis de conclure que $9 \%$ des affiliés à l'assurance de base d'Helsana ont eu au moins une prescription d'antidépresseurs en 2016 (110000 bénéficiaires sur un total de 1,2 million d'assurés AOS d'Helsana). Une estimation prudente d'Helsana table sur environ 730000 bénéficiaires de prescription d'antidépresseurs en Suisse (sur la base de la part de marché de 15\% détenue par Helsana).

Le rapport sur les médicaments 2017 d'Helsana confirme que les troubles mentaux constituent un facteur de coûts important [4]. Bien que le coût absolu des psychostimulants ait légèrement diminué ces cinq dernières années, les prescriptions de psychostimulants ont augmenté de plus de 10\% entre 2013 et 2016 pour atteindre plus de 4,2 millions.

A cela s'ajoute que plus de $60 \%$ des coûts relèvent du secteur hospitalier, lors de traitements en clinique (cf. fig. 2). Il existe donc un potentiel d'économie en

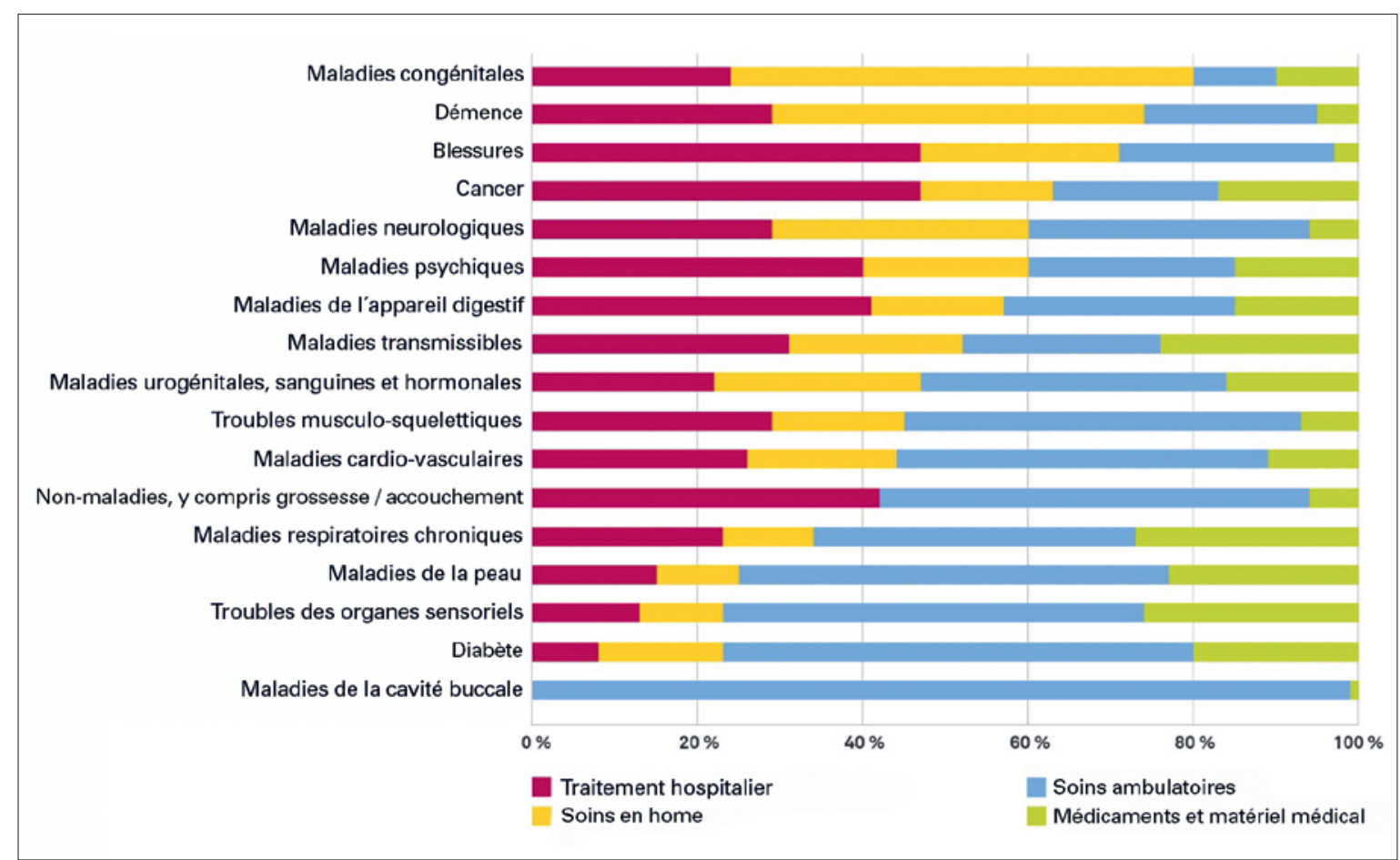

Figure 2: Parts des différents types de traitement dans les coûts des groupes de maladies. 
prescrivant davantage de traitements en ambulatoire. Il est surprenant de voir que la démence n'a pas un impact démesuré. Même si cette maladie est régulièrement pointée du doigt comme celle qui fait augmenter les coûts de la santé, les troubles de la démence ne coûtent pas plus de 1,1 milliard de francs par an, soit moins de deux pour cent $(1,7 \%)$ de l'ensemble des coûts de la santé. Ils se trouvent au même niveau que les troubles des organes sensoriels et les maladies de la peau. D'une part, cette maladie n'est pas aussi fréquente que beaucoup le croient et, de l'autre, le traitement de nombreux patients est comparativement bon marché pour le système de santé car une partie de la prise en charge est assumée par les proches.

\section{Le cancer à la septième place}

Alors que le cancer est la deuxième cause de mortalité, il occupe, en termes de dépenses, la septième place avec $6 \%$ de l'ensemble des coûts. Ce faible pourcentage s'explique d'un côté par une durée de traitement relativement courte. Comparativement, le laps de temps entre le diagnostic et le décès est souvent court, notamment pour les formes de cancer difficiles à traiter (par ex. cancer du poumon). De l'autre, le nombre de personnes atteintes d'un cancer est nettement plus faible que pour les maladies cardio-vasculaires ou les troubles musculo-squelettiques. Comme les progrès de la médecine et quelques nouveaux (et chers) médicaments permettent de prolonger l'espérance de vie de nombreux patients, le cancer devient de plus en plus une maladie chronique [5].

\section{Les soins intégrés augmentent la qualité et l'efficience}

L'étude montre que les maladies chroniques non transmissibles avec une forte prévalence prédominent dans les dépenses de santé en Suisse. Les maladies plus souvent mortelles comme de nombreuses formes de cancer constituent, à l'inverse, une part comparativement plus faible de l'ensemble des coûts.

Face à ces conclusions, la question se pose de savoir comment organiser la prise en charge de manière plus efficiente dans notre pays afin de freiner la hausse des coûts. Précisément pour les maladies chroniques comme les maladies cardio-vasculaires, le diabète et les douleurs de dos, les soins intégrés (Managed Care) pourraient fortement contribuer à améliorer la qualité et l'efficience de la prise en charge médicale [6].

\section{Références}

1 Wieser S, Riguzzi M, Pletscher M, Huber CA, Telser H, Schwenkglenks M. How much does the treatment of each major disease cost? A decomposition of Swiss National Health Accounts. Eur J Health Econ. 2018. https://doi.org/10.1007/s10198-018-0963-5.

2 Office fédéral de la santé publique (OFSP). https://www.bag.admin. ch/bag/de/home/themen/mensch-gesundheit/nichtuebertragbare-krankheiten/herz-kreislauf-erkrankungen.html

3 Enquête suisse sur la santé 2012, Office fédéral de la statistique (2013). https://www.bfs.admin.ch/bfs/fr/home/statistiques/sante/ enquetes/sgb.html

4 Helsana. Rapport sur les médicaments en Suisse 2017; p. 191 (en allemand). https://www.helsana.ch/docs/arzneimittelreport-2017.pdf

5 https://www.krebsgesellschaft.de/onko-internetportal/basis-informationen-krebs/basis-informationen-krebs-allgemeine-informationen/krebs-bald-eine-chronische-erkr.html

6 Huber CA, Reich O, Früh M, Rosemann T. Effects of Integrated Care on Disease-Related Hospitalisation and Healthcare Costs in Patients with Diabetes, Cardiovascular Diseases and Respiratory Illnesses: A Propensity-Matched Cohort Study in Switzerland. Int J Integrated Care. 2016;16(1):11. 